\title{
Nachhaltige Entwicklung in einer Gesellschaft des Umbruchs - Zur Einführung
}

\section{Birgit Blättel-Mink und Thomas Hickler}

\section{Zusammenfassung}

Noch nie waren Menschen global so vernetzt, und Informationen wurden so schnell um den Globus ausgetauscht wie heute. Dies führt zu Innovationen und vielen neuen Möglichkeiten. Der schnelle Wandel birgt jedoch auch Gefahren für Mensch und Natur. Dazu zählen der globale Verlust an Biodiversität und die irreversible Erderwärmung, und auch die akute Krise durch die CoronaPandemie ist ein Symptom derartiger Gefahren. Der Wissenschaftliche Beirat der Bundesregierung Globale Umweltveränderungen (WBGU 2011) und der Weltbiodiversitätsrat (IPBES 2019) fordern eine tiefgreifende gesellschaftliche Transformation in Richtung Nachhaltigkeit. Dafür braucht es auf der Ebene der Forschung mehr inter- bzw. transdisziplinäre Ansätze und damit auch neue Entwicklungen an den Universitäten. In dieser Einleitung fassen wir kurz die Beiträge von Kolleg*innen an der Goethe-Universität zu diesem Buch zusammen, welche sich aus ganz unterschiedlichen Perspektiven mit Nachhaltigkeit befassen.

\footnotetext{
B. Blättel-Mink ( $\square)$

Institut für Soziologie der Goethe-Universität Frankfurt am Main, Frankfurt am Main, Deutschland

E-Mail: b.blaettel-mink@soz.uni-frankfurt.de

T. Hickler

Senckenberg Biodiversitäts- und Klima Forschungszentrum (BiK-F) und Institut für Physikalische Geographie der Goethe-Universität in Frankfurt am Main, Frankfurt am Main, Deutschland

E-Mail: thomas.hickler@senckenberg.de
} 
Dass die wissenschaftliche Befassung mit dem Thema Nachhaltige Entwicklung sich nicht auf eine Disziplin beschränkt, wird bereits im 1972 erschienen Bericht des Club of Rome („Limits of Growth“; Meadows et al. 1972) deutlich gemacht. Nicht nur setzte und setzt sich der Club of Rome aus Vertreter*innen unterschiedlicher Disziplinen zusammen, sondern wird auch im damaligen Bericht betont, dass gesellschaftliche Herausforderungen, beispielsweise die Versorgung der Bevölkerung mit Nahrungsmitteln, immer auch eine ökonomische Komponente aufweisen, dass sie jedoch auch abhängig sind vom individuellen Konsum sowie von den vorhandenen ökologischen Ressourcen.

In Zeiten eines rasanten globalen Wandels ist das Thema Nachhaltigkeit aktueller denn je. Noch nie waren Menschen global so vernetzt, und Informationen wurden so schnell um den Globus ausgetauscht wie heute. Dies führt zu zahlreichen Innovationen und vielen neuen Möglichkeiten, aber der schnelle Wandel birgt auch Gefahren. Wir greifen so stark in das Erdsystem ein, dass man zunehmend von einem durch den Menschen geprägten Erdzeitalter spricht, dem „Anthropozän“, insbesondere seitdem unsere Aktivitäten das globale Klima verändern (Crutzen 2002; IPCC 2013; Steffen et al. 2015). Der Mensch hat heute bspw. mehr als 1/3 der Landfläche für seine Zwecke transformiert (Steffen et al. 2015; IPCC 2019), und wir haben den Stickstoffeintrag in Ökosysteme im Vergleich zum natürlichen Zustand verdoppelt (Fowler et al. 2013). In Europa wurden die ökonomischen Kosten der Überdüngung, bspw. durch Nitrat im Grundwasser, auf 70 bis 320 Mrd. EUR pro Jahr geschätzt, d. h. wahrscheinlich höher als die Erträge der Landwirtschaft durch Düngung (Sutton et al. 2011). Die biologische Vielfalt des Planeten ist stark rückläufig (Pimm et al. 2014; Newbold et al. 2015) ${ }^{1}$. Laut einer Schätzung des Weltbiodiversitätsrats (,Intergovernmental Science-Policy Platform on Biodiversity and Ecosystem Services“, IPBES) sind ca. eine Million von acht Millionen Tier- und Pflanzenarten vom Aussterben bedroht (IPBES 2019). Durch den Verlust an Arten und Ökosystemen verlieren wir global Ökosystemleistungen im Wert von mehreren Billionen Euro jährlich (Costanza et al. 2014). Außerdem führt die Akkumulation einer Vielzahl von Umweltchemikalien in Gewässern, Böden, Lebensmitteln und Menschen zu kaum abzuschätzenden Gefahren für unsere Gesundheit (Landrigan et al. 2016).

Ein „Weiter so“ ist vor dem Hintergrund dieser rasanten Dynamik keine Option. Der Klimawandel macht dies besonders deutlich. Pessimistische Annahmen in Bezug auf unsere zukünftigen globalen Treibhausgasemissionen würden nach aktuellen Klimasimulationen wahrscheinlich eine globale Erwärmung von knapp 2,5 Grad Celsius bis zu 5,5 Grad Celsius bedeuten, mit erheblich höheren

\footnotetext{
${ }^{1}$ https://www.iucnredlist.org
} 
Veränderungen in einigen Regionen (Szenario RCP8.5, IPCC 2013). Eine 5 Grad wärmere Welt kann man sich kaum vorstellen. Zum Vergleich sei daran erinnert, dass Deutschland zum Höhepunkt der letzten Eiszeit, als es im globalen Durchschnitt 4-7 Grad kälter war, von einer eiszeitlichen Kältesteppe bzw. Eis bedeckt war. Die Folgen eines starken menschgemachten Klimawandels reichen von einer Umverteilung der globalen Wasserressourcen bis zu einem langfristig (über Hunderte von Jahren) mehrere Meter höheren Meeresspiegel (IPCC 2014; Clark et al. 2016). Außerdem können wir nicht ausschließen, dass schwer zu quantifizierende positive Rückkopplungsmechanismen dazu führen, dass der Klimawandel extremer wird als von unseren Klimamodellen projiziert (Steffen et al. 2018). Schließlich ist der oft propagierte Widerspruch zwischen wirtschaftlicher Entwicklung und einer Verringerung unserer Treibhausgasemissionen ein Mythos. Die „Global Commission on the Economy and Climate“ schätzt, dass entschlossenes Handeln zur Begrenzung des Klimawandels bis $2030 \mathrm{zu}$ einem direkten wirtschaftlichen Gewinn von 26 Billionen US-\$ führen könnte, verglichen mit einem ,Weiter so“ Szenario. ${ }^{2}$

Viele Szenario-Studien deuten darauf hin, dass es technisch möglich und ökonomisch sinnvoll ist umzusteuern (z. B. Popp et al. 2017; Grubler et al. 2018; IPCC 2018), wobei die nötigen Veränderungen teilweise schon im Gange sind, bspw. ein, global gesehen, massiver Ausbau der erneuerbaren Energien (IEA 2017). Im letzten Jahrzehnt sind die Kosten für Solarstrom um $80 \%$ gesunken, und neue Anlagen für Stromerzeugung nutzen bereits heute $\mathrm{zu}$ mehr als $50 \%$ erneuerbare Energiequellen. Die Stromerzeugung aus Wind und Sonne verdoppelt sich zurzeit alle 4 Jahre (Figueres et al. 2018). Dementsprechend sinken die Emissionen von Kohlendioxid, dem wichtigsten durch den Menschen emittierten Treibhausgas, in Europa seit ca. 30 Jahren und in den Vereinigten Staaten von Amerika seit ca. 10 Jahren. Auch in China sind sie in den letzten 10 Jahren trotz starken Wirtschaftswachstums kaum noch gestiegen. Aufgrund von steigenden Emissionen in anderen Weltregionen, z. B. in Indien, haben die globalen Emissionen jedoch bis vor Kurzem weiter zugenommen (Friedlingstein et al. 2019). ${ }^{3}$

Und dann kam die Corona-Pandemie. Im April 2020 hat die Internationale Energieagentur (IEA) geschätzt, dass die Kohlendioxid-Emissionen 2020 um ca. $8 \%$ fallen könnten, aber man erwartet, dass dieser Rückgang nur vorübergehend ist. Die Pandemie zeigt jedoch vor allem, wie vernetzt und - nicht zuletzt deshalb - wie verwundbar Gesellschaften sind. Und sie zeigt, dass wir

\footnotetext{
2 https://newclimateeconomy.report

${ }^{3}$ https://www.globalcarbonproject.org
} 
im nationalen wie im globalen Kontext entschieden handeln können und müssen. Klimawandel und der Verlust an biologischer Vielfalt sind jedoch im Gegensatz zu Covid-19 eine Bedrohung für menschliches Wohlergehen über Jahrhunderte.

Um die für eine Begrenzung des Klimawandels und den Erhalt unserer natürlichen Lebensgrundlagen nötigen sozioökonomischen Transformationen zu erreichen, muss erheblich mehr passieren als bisher (IPCC 2018; IPBES 2019). Wir müssen es wagen, eine andere Welt zu denken, und wir müssen die Schranken in unseren Köpfen hinterfragen. Eine interdisziplinäre Nachhaltigkeitsforschung kann hierzu wichtige Beiträge liefern.

Über viele Jahre, und bedingt durch die Definition der Vereinten Nationen, galten drei Dimensionen und damit auch drei Disziplinen bzw. Fächergruppen als für die Forschung zu Nachhaltigkeit zentral: die Naturwissenschaften, die Wirtschafts- und die Sozialwissenschaften. Die Politik galt schließlich zuständig dafür, dass Programme für die Forschung zu Nachhaltiger Entwicklung aufgelegt wurden, und dass die gewonnenen Erkenntnisse Eingang in politische Strategien fanden. Als Vertreter*innen der GRADE (Goethe Graduate School) bzw. von GRADE-Sustain (Graduierten Zentrum für Nachhaltige Entwicklung) in Frankfurt am Main im Jahre 2015 eine knapp zweijährige Vortragsreihe zum Thema Nachhaltige Entwicklung organisierten, um damit die Forschungsperspektiven, die an der Goethe-Universität, aber auch an außeruniversitären Forschungseinrichtungen (Senckenberg Gesellschaft für Naturforschung und ISOE - Institut für sozial-ökologische Forschung) in Frankfurt am Main, existieren, miteinander ins Gespräch zu bringen, wurde schnell deutlich, dass ein Fokus auf die drei Fächergruppen deutlich zu eng ist. Auch wenn dieser Band die Vielfalt der Disziplinen und ihre Befassung mit Nachhaltiger Entwicklung nicht gänzlich abbildet, finden sich doch neben den „klassischen“ Disziplinen (Ökologie, Politikwissenschaft, Soziologie, Humangeografie), die Kunstwissenschaften, die Theologie und die Philosophie.

Dass sich eine Volluniversität wie die Goethe-Universität dem Thema Nachhaltige Entwicklung aus unterschiedlichen Perspektiven zuwendet, verweist auch auf die institutionelle Wahrnehmung der Verantwortung beziehungsweise der gesellschaftlichen Einbettung von Universitäten. Das bedeutet noch nicht, dass die Goethe-Universität sich in Richtung einer transformativen Hochschule wandelt (vgl. Schneidewind und Singer-Brodowski 2014), aber es bedeutet, dass Forschung, die sich mit den Bedingungen einer gesellschaftlichen Transformation in Richtung Nachhaltigkeit im Sinne des Wissenschaftlichen Beirats der Bundesregierung Globale Umweltveränderungen (WBGU 2011) befasst, an dieser Universität durchaus einen Platz hat. Aktuell hat sich dort, nicht zuletzt aufgrund 
der Initiative einer Gruppe von sich für Nachhaltigkeit einsetzender Studierender und mit Unterstützung des Präsidiums, eine Arbeitsgruppe Nachhaltigkeit gegründet, die sich neben konzeptioneller Aspekte mit ganz konkreten Herausforderungen einer nachhaltigen Universität beschäftigen: in den Felder des Bauens, der Ernährung, der Mobilität und der Energie.

Im Folgenden wird der bunte Strauß der Nachhaltigkeitsforschung, wie er sich in dieser Publikation entfaltet, kurz vorgestellt.

Petra Döll (,Risiken des Klimawandels: Wie kann man mit den vielfältigen Unsicherheiten bei Risikobewertung und Anpassung an den Klimawandel umgehen?"), Hydrologin mit einem Fokus auf Wasser im globalen Kontext und auf partizipative Strategien transdisziplinärer Forschung, thematisiert die Wahrnehmung von Risiken des anthropogenen Klimawandels (KW) als Prozesse, die mit ganz unterschiedlichen Formen der Unsicherheit behaftet sind. Wenn heterogene Stakeholder und Wissenschaftler*innen unterschiedlicher Disziplinen und Wissenschaftskulturen aufeinandertreffen, um gemeinsam Risiken auszumachen und zu bewerten, so Dölls Argument, geht es nicht nur um ontologische Unsicherheiten, sondern auch um epistemische und linguistische Unsicherheiten sowie um Uneindeutigkeiten. „Wir können mögliche zukünftige Entwicklungspfade nennen, aber keine gesicherten Aussagen darüber machen, welche Pfade jeweils wahrscheinlicher sind als andere. Diese Art von epistemischer Unsicherheit kann als „tiefe“ Unsicherheit bezeichnet werden [...]. Aufgrund dieser 'tiefen' Unsicherheit wird bei der Bewertung von KW-Risiken statt mit probabilistischen Vorhersagen (,ein bestimmtes Ereignis wird mit einer Wahrscheinlichkeit von x auftreten") mit Szenarien zukünftiger Treibhausgase und zukünftiger sozioökonomischer Bedingungen gearbeitet.“ (Döll in diesem Band S. 20f)

Der Politikwissenschaftler Darrel Moellendorf („Climate Change, Policy, and Justice“), beschäftigt sich mit Fragen der intra- und intergenerationalen Gerechtigkeit im Kontext des Klimawandels. Er konstatiert in seinem Beitrag, dass eine gerechte Verteilung von Kosten über die Generationen und zwischen dem globalen Norden und Süden, eine normative Frage ist, und nur möglich wird, wenn sich die nationalen Politiken wie auch die Institutionen entsprechend anpassen und sich aktiv für eine Verlangsamung des Klimawandels einsetzen und dafür auch zu internationalen Kompensationsleistungen bereit sind. Dafür konfrontiert er in seinem Beitrag die beiden vor allem vom IPCC - Intergovernmental Panel of Climate Change - genannten Lösungswege in Richtung Nachhaltiger Entwicklung: Mitigation - Bekämpfung der Ursachen des Klimawandels und Adaptation - Anpassung des menschlichen Handelns an die Folgen des Klimawandels - und diskutiert diese entlang der Konsequenzen für intergenerationale Gerechtigkeit auf der einen und globale Gerechtigkeit auf der anderen Seite. Er schlussfolgert: 
„The right to sustainable development requires that rich states provide support for adaptation and compensation policies in poor countries in order that the latter may establish protection against the threats of climate change. Justice makes demands. In the case of climate change, responding adequately to these demands requires international cooperation in order to affect a rapid transition to renewable energy production and consumption and to safeguard conditions in which continued progress in human development can be made." (Moellendorf in diesem Band, S. 42).

Die Biologinnen Karen Hahn und Anna Leßmeister (,Sustainable use of savanna vegetation in West Africa in the context of climate and land use change") untersuchen, wie Menschen in Savannen die Natur nutzen. Menschen in ländlichen Regionen in Afrika tun dies auf vielfältige Weise und sind dabei extrem abhängig von Ökosystemleistungen. Außerdem sind sie besonders stark von den Folgen des Klimawandels betroffen, weil alternative Einkommensquellen sowie Ressourcen für Anpassung kaum vorhanden sind. Allerdings gibt es kaum Daten dazu, welche Arten wie genutzt und wertgeschätzt werden. Die Forschung von Hahn und Leßmeister zeigt, dass in Savannen eine Vielzahl von Arten genutzt werden und erheblich zum Einkommen beitragen. Diese Nutzung erscheint oft nicht in regionalen oder globalen Landnutzugskarten, sodass der irrtümliche Eindruck entstehen kann, dass die Ökosysteme nicht genutzt werden und damit für andere Nutzungstypen wie bspw. Bioenergieplantagen zur Verfügung stehen. Die Autorinnen quantifizieren diese Leistungen und tragen damit zu für planerischen und entwicklungspolitischen Prozessen bei für die Anpassung an den Klimawandel. Sie verweisen auch darauf, dass die Bedürfnisse und Argumente der ländlichen Bevölkerung in der großräumigen Planung oft nicht berücksichtigt werden. „Thus, transdisciplinary research approaches are required which comprise collaboration between multiple scientific disciplines and the integration of extra-scientific knowledge of practitioners and stakeholders throughout the entire research process [...].“ (Hahn und Leßmeister in diesem Band, S. 47).

Robert Pütz und Antje Schlottmann („Umkämpfte Nachhaltigkeit - vergessene Leiblichkeit. Der Fall der Wildpferde in Namibia“) vom Institut für Humangeografie der Goethe-Universität untersuchen in ihrem Beitrag den Umgang mit Wildpferden in einem Nationalpark Namibias. Sie bezeichnen ökologische Nachhaltigkeit mit Ernesto Laclau (2002) als „leeren Signifikanten“, der diskursiv durch spezifische, häufig konkurrierende Naturvorstellungen immer wieder neu gefüllt wird. Mit einer Diskursanalyse können dann folgende Fragen beantwortet werden: „Welche Tiere haben im Kampf um die größte symbolische Bedeutung den Vorzug und „dürfen“ Namibias Naturlandschaft repräsentieren? Und das heißt letztlich auch: Welche Tiere dürfen überleben und welche nicht?"“ (Pütz 
und Schlottmann in diesem Band S. 73). Der Befund, dass eine solche Perspektive allein das Verständnis des Umgangs mit bedrohten Arten nicht adäquat nachvollziehbar macht, führt in der weiteren Argumentation zum AssemblageKonzept in der Lesart des Neuen Materialismus. Mit ihm wird die aktive Handlungsmacht (agency) der Wildtiere im Mensch-Wildpferd-Netzwerk in die Betrachtung einbezogen. Mit dem Konzept von Zwischenleiblichkeit (intercorporeality) nach Maurice Merleau-Ponty (1966), welches auch die leibgebundenen Interaktionen und Aneignungen der Beteiligten erfasst, ergibt sich schließlich eine wissenschaftliche Analyse von zwischenleiblichen Situationen, in denen sich die Grenzen zwischen Mensch und Tier ebenso verflüssigen, wie die zwischen Diskurs und leiblicher Praxis. Konzepte wie Zwischenleiblichkeit eröffnen damit nicht-dualistisch angelegte Zugänge, mit denen z. B. die Konsequenzen verinnerlichter und veräußerter dualistischer Rationalitäten kritisch betrachtet werden können und die insofern post-dualistische Forderungen erfüllen. Sie ,eignen sich aber auch als Basis für eine andere Ontologie von Nachhaltigkeit, die derart aus Interaktionen gewonnenes Wissen akzeptiert" (Pütz und Schlottmann in diesem Band, S. 92).

Bruno Streit („,Der Mensch und der Rhein“), Emeritus am Institut für Ökologie, Evolution und Diversität der Goethe-Universität mit einem Fokus auf die Fauna von Fließgewässern, beschreibt den Rhein in einem weiten Bogen als historisch, territorial wie ökologisch, sich wandelndes Wasserökosystem, als Bezugsund Orientierungspunkt für Anwohner*innen, Besucher*innen, aber auch als Einkommensquelle für Vertreter*innen ganz unterschiedlicher Berufe. Er unterscheidet ausgewiesene Naturschutzgebiete des Rheins, die für naturverbundene Menschen ein Erholungsgebiet darstellen, und Orte der „Rheinromantik“ wo „rheinische Fröhlichkeit“ gelebt wurde und noch wird. „Geplant worden ist diese facettenreiche und in den Regionen auch identitätsfördernde Gemütswirkung nie, sondern sie ist das mehr beiläufige Produkt der historischen und politischen, gesellschaftlichen und kulturellen sowie der wirtschaftlichen und wasserbaulichen Geschichte. Was der Rhein nur noch in Resten bieten kann, ist ein ursprünglicher Flusscharakter, denn er ist hydrologisch gebändigt, biologisch umgekrempelt und gesellschaftlich vermarktet." (Streit in diesem Band, S. 100). Es wundert denn auch nicht, dass Streit In seinem äußerst kenntnisreichen geschriebenen Beitrag am Ende resümiert: ,Die Rheingeschichte lehrt darüber hinaus auch, dass Umweltveränderungen stark durch politische, wirtschaftliche und gesellschaftliche Ziele und Zwänge hervorgerufen werden und dass ein gleichsam museales Konservieren von belebten Naturausschnitten illusorisch ist. Jede Generation formuliert neue Ansprüche und Ziele und ist sich auch nie über alle Folgen von 
vornherein im Klaren - oder nimmt sie bewusst in Kauf." (Streit in diesem Band, S. 116 f.).

Die Soziologin Birgit Blättel-Mink (,Nachhaltige Entwicklung als Strategie der Völkergemeinschaft zur Überwindung der „Grenzen des Wachstums“. Ein kritisch-historischer Abriss"), Professorin am Institut für Soziologie der GoetheUniversität, geht recht weit zurück in die Geschichte des Leitbildes Nachhaltige Entwicklung und skizziert in einem historischen Abriss die zentralen politischen, gesellschaftlichen und wissenschaftlichen Lesarten der Nachhaltigkeit. Sie diskutiert den Erfolg der Nachhaltigkeitsstrategien der Völkergemeinschaft kritisch und wirft schließlich einen Blick auf die Rolle der Wissenschaft für die Durchsetzung einer nachhaltigen Entwicklung - vor allem im Übergang zum Anthropozän. Sie verweist dabei auf das Gutachten des WBGU, in welchem die Autor*innen sowohl eine Stärkung der Transformationsforschung - Wege in Richtung Nachhaltigkeit - als auch der transformativen Wissenschaft einfordern. Letztere wird von Uwe Schneidewind wie folgt definiert: ,Transformative Wissenschaft bezeichnet eine Wissenschaft, die gesellschaftliche Transformationsprozesse nicht nur beobachtet und von außen beschreibt, sondern diese Veränderungsprozesse selber mit anstößt und katalysiert und damit als Akteur (teilnehmender Beobachter) von Transformationsprozessen über diese Veränderungen lernt." (Schneidewind 2015, Blättel-Mink in diesem Band, S. 135). Damit steht die Wissenschaft der Zukunft vor großen Herausforderungen.

Thomas Jahn (,,Transdisziplinäre Nachhaltigkeitsforschung - Methoden, Kriterien, gesellschaftliche Relevanz"), einer der Mitbegründer des Instituts für sozial-ökologische Forschung in Frankfurt am Main (ISOE) und langjähriger wissenschaftlicher Geschäftsführer dieser Einrichtung, skizziert einen transdisziplinären Ansatz der Nachhaltigkeitsforschung, in dem nicht nur die Forschung jenseits disziplinärer Grenzen durchgeführt wird, sondern auch das beforschte Phänomen gesellschaftlich verankert ist und wo die Ergebnisse der Forschung, die in der Regel gemeinsam mit Vertreter*innen gesellschaftlicher Gruppen durchgeführt werden, in die Gesellschaft hinein getragen werden. „Das am ISOE entwickelte Modell eines idealtypischen, transdisziplinären Forschungsprozesses [...] wurde in zahlreichen Forschungsprojekten auch außerhalb des ISOE praktisch erprobt (Jahn et al. 2012). Es geht von der Grundannahme aus, dass gesellschaftliche Probleme in der Regel auf Lücken im verfügbaren wissenschaftlichen Wissen verweisen. Durch die damit implizierte Verknüpfung gesellschaftlicher Probleme mit originären wissenschaftlichen Problemen wird es möglich, Beiträge zum gesellschaftlichen $u$ d wissenschaftlichen Fortschritt als epistemisches Ziel einer einzigen Forschungsdynamik zu betrachten. In diesem Ansatz ist damit die spannungsreiche Frage nach der Relevanz von Forschung allgemein und von 
Nachhaltigkeitsforschung im Besonderen aufgehoben." (Jahn in diesem Band, S. 147 f.). Jahn identifiziert in seinem Beitrag methodische Herausforderungen und benennt Qualitätskriterien transdisziplinärer Nachhaltigkeitsforschung, die sich im aktuellen Wissenschaftssystem vielfältigen institutionellen constraints gegenübergestellt sieht, die es bislang noch von Fall zu Fall zu überwinden gilt.

Rosa Sierra (,Transformation, contestation and normativity. Learning from actors and socio-political engagements in transformative science“), Philosophin und Gruppenleiterin für das Teilprojekt Nachhaltigkeit des deutsch-französisichen Kooperationsprojekts „Saisir l'Europe“, welches am Institut für Philosophie der Goethe-Universität angesiedelt ist, befasst sich in ihrem Beitrag mit einer Sichtweise auf Transformationsforschung, wie sie der WBGU fordert. Als zentrale Herausforderung des TRANSFORM Verständnisses von „Saisir l'Europe“ wird die Spannung zwischen einer system- und einer akteursorientierten Perspektive gesehen. Sierra, bzw. ein Netzwerk deutsch-französischer Wissenschaftler*innen, welches sich mit Transformationsforschung beschäftigt, konstatiert ein Paradoxon: „However, the research of agency towards transformation shows what appears to be a paradoxical dynamic in transformations towards sustainability: the goal of sustaining the states, structures or practices envisaged as the aim of transformations seems to preclude further transformations." (Sierra in diesem Band, S. 170). Anders formuliert: wie umgehen mit dem Widerspruch von Erhalten und Transformieren? Für eine Lösung greift Sierra auf das Konzept des ISOE, konkret auf einen Beitrag von Thomas Jahn (2013) zurück, in dem dieser argumentiert, dass es in der Nachhaltigkeitswissenschaft darauf ankommt, solche Prozesse zu identifizieren, die Nachhaltigkeit ermöglichen, und zu bestimmen, welche Bedingungen gegeben sein müssen, damit diese Prozesse erhalten bleiben können. „From this point of view, the continuity of a system's capacity for further development rather than the continuation of given states is the key feature of the sustainability model." (Sierra in diesem Band, S. 171). In ihrem Beitrag konkretisiert sie dieses Verständnis beispielhaft für Projekte von „Saisir l'Europe“.

Im Rahmen einer am ISOE angesiedelten BMBF-geförderten Nachwuchsgruppe arbeiten die beiden Leiterinnen, die Humangeografin Johanna Kramm und die Ökotoxikologin Carolin Völker (,Wie ist ein nachhaltiger Umgang mit Plastik möglich? Eine Vorstellung der inter- und transdisziplinär arbeitenden Nachwuchsgruppe ,PlastX“"), disziplinenübergreifend zusammen, um gemeinsam mit Doktorand*innen unterschiedlicher disziplinärer Ausrichtungen umfassende Erkenntnisse zum Thema Plastik in der Umwelt zu erhalten und gesellschaftliche Schritte in Richtung Reduktion von Mikroplastik, vor allem in Wasserökosystemen, zu entwickeln. In welcher Weise Mikroplastik ein systemisches Risiko darstellt, beschreiben die Autorinnen: „Plastik ist günstig herstellbar, gut formbar, 
leicht und beständig und bietet deshalb viele Vorteile gegenüber anderen Materialien. Letztendlich sind es jedoch genau diese Vorteile, die zu den unerwünschten Effekten in der Umwelt führen - die massenhafte Verwendung führt zu einem hohen Abfallaufkommen und zum Eintrag in die Umwelt und die Beständigkeit des Materials setzt sich auch in der Umwelt fort, was bedeutet, dass die meisten Kunststoffe dort akkumulieren und kaum oder nur sehr langsam abgebaut werden. Diese Ambivalenz verdeutlicht, dass es sich bei dem Thema nicht um ein rein wissenschaftliches, sondern um ein komplexes lebensweltliches Problem handelt.“ (Kramm und Völker in diesem Band, S. 177). In ihrem Beitrag stellen die Autorinnen die transdisziplinäre Vorgehensweise in der Nachwuchsgruppe sowie die einzelnen Projekte vor.

Der Sozialethiker Johannes Hoffmann (,Die Forschungsgruppe EthischÖkologisches Rating an der Goethe-Universität. Forschungsergebnisse und ihre Wirkung in Wirtschaft und Politik") stellt in seinem Beitrag die Arbeit der Forschungsgruppe Ethisch-Ökologisches Rating an der Goethe-Universität dar. Die Grundfragen dieser interdisziplinären Forschungsgruppe, die seit Oktober 2018 an das Weltethos Forschungsinstitut an der Universität Tübingen angegliedert ist, formuliert er wie folgt: ,a) Welche Bedeutung hat die Allgemeine Menschenrechtserklärung für die Realisierung menschenwürdiger Lebensbedingungen in unserer und in uns fremden Kulturen? b) Welche Konsequenzen ergeben sich daraus für die Gestaltung einer Marktwirtschaft, dass durch sie die Erhaltung der Substanz ökonomischer, ökologischer, sozialer und kulturelle Ressourcen für uns und künftige Generationen gesichert werden kann?" (Hoffmann in diesem Band, S. 198). Dafür hat die Gruppe einen Indikator entwickelt, der in der Folge auch Eingang fand in Wirtschaftsunternehmen, der jedoch angesichts der Wachstumsund Wettbewerbsorientierung der Wirtschaft, so Hoffmann, das damit verknüpfte Ziel einer deutlich nachhaltigeren Wirtschaft im Sinne von sozialer und ökologischer Nachhaltigkeit nicht erreicht hat. In der Konsequenz fordert die Gruppe, und das führt Hoffmann in seinem Beitrag aus, eine ethisch fundierte Änderung des deutschen Wettbewerbsrechts. „Unsere Gesetze verhindern den Ressourcenschutz! Nachhaltiger Wettbewerb muss einklagbar werden!“ (Hoffmann in diesem Band, S. 209).

Verena Kuni („Ars Longa. Kunst und Nachhaltigkeit“), Professorin für Visuelle Kultur am Institut für Kunstpädagogik der Goethe-Universität, diskutiert in ihrem Beitrag die Rolle der Kunst für Nachhaltige Entwicklung. Sie nimmt den Aphorismus des Hippokrates „Vita brevis, ars longa“ zum Anlass, darüber nachzudenken, in welcher Weise die Kunst, die aktuell nicht gerade dafür steht, konservierbare Werkstoffe zu benutzen, Beiträge zur Nachhaltigkeit leistet. Sie greift dafür einige Beispiele heraus, vor allem die Arbeit „7000 Eichen“ von 
Joseph Beuys, der nicht nur Kunst schaffen, sondern auch gesellschaftlich etwas bewegen wollte. Kuni findet für eine Kunst bzw. eine Kultur, die sich in diesen Kontext einordnet, folgende Definition: „... Bilder zu schaffen, die - wortwörtlich nachhaltig - zum Denken und Handeln anregen". (Kuni in diesem Band, S. 219). Nach Beuys entstanden so viele, ganz unterschiedliche Projekte, die, vor allem Kunst und Wissenschaft miteinander verknüpften, wo die Kollaboration von Kunst, Natur und Gesellschaft dazu beitragen soll, deutliche Schritte in Richtung Nachhaltigkeit zu gehen. Kuni verweist unter anderem auf Bienen-Projekte, vor allem in städtischen Räumen, darunter Frankfurt am Main. Sie schließt ihren Beitrag mit einer Beobachtung: „Heute hingegen scheint es nahezu selbstverständlich, dass Künstler*innen nicht allein aus dem Atelier heraus operieren, sondern mit Projekten direkt in die Öffentlichkeit gehen - und dabei etwa auch unter den Vorzeichen der Kunst Bäume pflanzen oder eine Stadtimkerei betreiben. Kunst, die kulturelle Bildung, ökologisches und soziales Engagement verknüpft, hat sich als zukunftsfähig erwiesen: Als , ars longa', die das Thema Nachhaltigkeit - und die mit ihm verknüpften Fragen und Komplexe - nicht nur aufgreift und in Bilder fasst, sondern direkt zum Handeln und Mittun einlädt." (Kuni in diesem Band, S. 240).

Abschließend diskutiert der Paläontologe Volker Mosbrugger (,Ökologischer Imperativ, Nachhaltigkeit, Planetare Grenzwerte und „One Health“ -Zielfunktionen für ein zukunftsfähiges Geoengineering“") mögliche Zielfunktionen bzw. wissenschaftliche Konzepte für ein Erdsystemmanagement. Diese reichen von den klassischen Nachhaltigkeitskonzepten bis zu planetaren Grenzen und einer Verknüpfung von menschlicher Gesundheit und der Gesundheit der Natur. Hierbei betont er, dass wir sogenanntes „Geo-engineering“, welches in der Gesellschaft oft kritisch gesehen wird, im Anthropozän sowieso betreiben, jedoch ohne die nötige globale Koordination, die notwendig wäre, um gemeinsame Ziele zu erreichen. Er vergleicht die enorme Herausforderung, der sich die Menschen gegenübersehen, mit der Entwicklung der Humanmedizin. „Die Entwicklung der Humanmedizin zu einer modernen, erfolgreichen Wissenschaft hat mehrere Jahrhunderte gebraucht. Die Entwicklung eines modernen Erdsystem-Managements im Sinne einer Heilkunde der Erde muss angesichts der anthropozänen Herausforderung deutlich schneller erfolgen." (Mosbrugger in diesem Band, S. 248).

Wir danken den Autorinnen und Autoren dieses Bandes für ihre informativen und weiterführenden Beiträge in Sachen Nachhaltigkeit. Wir danken insbesondere Frau Dr. Henrike Becker, Referentin für Natur- und Lebenswissenschaften bei GRADE (Goethe Research Academy for Early Career Researchers) für Ihre Initiative, die Vorträge zu verschriftlichen und für Ihre Geduld in dieser Sache. Ohne sie wäre dieser Band mit Sicherheit nicht zustande gekommen. Wir hoffen, 
dass das Engagement der Goethe-Universität in dieser Sache sowohl in Forschung als auch in Lehre in Zukunft noch stärker sein wird.

„Wer [...] wissenschaftlich und/oder künstlerisch im Feld arbeitet, wird dies kaum von Konjunkturen abhängig machen. Vielmehr ist bereits der Frage nach Nachhaltigkeit eine Zeitperspektive eingeschrieben, die auf eine langfristige Beschäftigung mit der Sache und auf die Ausdauer aller Beteiligten setzt." (Kuni in diesem Band, S. 216).

\section{Literatur}

Clark, P. U., J. D. Shakun, S. A. Marcott, A. C. Mix, M. Eby, S. Kulp, A. Levermann, G. A. Milne, P. L. Pfister, B. D. Santer, D. P. Schrag, S. Solomon, T. F. Stocker, B. H. Strauss, A. J. Weaver, R. Winkelmann, D. Archer, E. Bard, A. Goldner, K. Lambeck, R. T. Pierrehumbert, und G.-K. Plattner. 2016. Consequences of twenty-first-century policy for multi-millennial climate and sea-level change. Nature Climate Change 6:360-369.

Costanza, R., R. de Groot, P. Sutton, S. van der Ploeg, S. J. Anderson, I. Kubiszewski, S. Farber, und R. K. Turner. 2014. Changes in the global value of ecosystem services. Global Environmental Change 26:152-158.

Crutzen, P. J. 2002. Geology of mankind. Nature 415:23-23.

Figueres, C., C. Le Quere, A. Mahindra, O. Bate, G. Whiteman, G. Peters, und D. Guan. 2018. Emissions are still rising: Ramp up the cuts. Nature 564:27-30.

Fowler, D., M. Coyle, U. Skiba, M. A. Sutton, J. N. Cape, S. Reis, L. J. Sheppard, A. Jenkins, B. Grizzetti, J. N. Galloway, P. Vitousek, A. Leach, A. F. Bouwman, K. Butterbach-Bahl, F. Dentener, D. Stevenson, M. Amann, und M. Voss. 2013. The global nitrogen cycle in the twenty-first century. Philosophical Transactions of the Royal Society B-Biological Sciences 368:20130164.

Friedlingstein, P., M. W. Jones, M. O’Sullivan, R. M. Andrew, J. Hauck, G. P. Peters, und S. Zaehle. 2019. Global carbon budget 2019. Earth System Science Data 11 (4):1783-1838.

Grubler, A., C. Wilson, N. Bento, B. Boza-Kiss, V. Krey, D. L. McCollum, N. D. Rao, K. Riahi, J. Rogelj, S. De Stercke, J. Cullen, S. Frank, O. Fricko, F. Guo, M. Gidden, P. Havlik, D. Huppmann, G. Kiesewetter, P. Rafaj, W. Schoepp, und H. Valin. 2018. A low energy demand scenario for meeting the 1.5 degrees $\mathrm{C}$ target and sustainable development goals without negative emission technologies. Nature Energy 3:515-527.

International Energy Agency (IEA). 2017. World energy outlook 2017. IEA publications. www.iea.org.

IPBES. 2019. Summary for policymakers of the global assessment report on biodiversity and ecosystem services of the intergovernmental science-policy platform on biodiversity and ecosystem services, Hrsg. S. Díaz, J. Settele, E. S. Brondízio, H. T. Ngo, M. Guèze, J. Agard, A. Arneth, P. Balvanera, K. A. Brauman, S. H. M. Butchart, K. M. A. Chan, L. A. Garibaldi, K. Ichii, J. Liu, S. M. Subramanian, G. F. Midgley, P. Miloslavich, Z. Molnár, D. Obura, A. Pfaff, S. Polasky, A. Purvis, J. Razzaque, B. Reyers, R. Roy Chowdhury, Y. J. Shin, I. J. Visseren-Hamakers, K. J. Willis, and C. N. Zayas, 56 pages. Bonn: IPBES secretariat. 
IPCC. 2013. Climate change 2013: The physical science basis. Contribution of working group I to the fifth assessment report of the intergovern- mental panel on climate change, Hrsg. T. F. Stocker, D. Qin, G.-K. Plattner, M. Tignor, S. K. Allen, J. Boschung, A. Nauels, Y. Xia, V. Bex and P. M. Midgley. Cambridge: Cambridge University Press.

IPCC. 2014. Climate change 2014: Mitigation of climate change. Contribution of working group III to the fifth assessment report of the intergovernmental panel on climate change, Hrsg. O. Edenhofer, R. Pichs-Madruga, Y. Sokona, E. Farahani, S. Kadner, K. Seyboth, A. Adler, I. Baum, S. Brunner, P. Eickemeier, B. Kriemann, J. Savolainen, S. Schlömer, C. von Stechow, T. Zwickel und J. C. Minx. Cambridge: Cambridge University Press.

IPCC. 2018. Global warming of 1.5 C. An IPCC special report on the impacts of global warming of $1.5 \mathrm{C}$ above pre-industrial levels and related global greenhouse gas emission pathways, in the context of strengthening the global response to the threat of climate change, sustainable development, and efforts to eradicate poverty, Hrsg. V. Masson-Delmotte, P. Zhai, H. O. Pörtner, D. Roberts, J. Skea, P. R. Shukla, A. Pirani, W. Moufouma-Okia, C. Péan, R. Pidcock, S. Connors, J. B. R. Matthews, Y. Chen, X. Zhou, M. I. Gomis, E. Lonnoy, T. Maycock, M. Tignor und T. Waterfield. Geneva: IPCC.

IPCC. 2019. Summary for policymakers. In Climate change and land: An IPCC special report on climate change, desertification, land degradation, sustainable land management, food security, and greenhouse gas fluxes in terrestrial ecosystems, Hrsg. P. R. Shukla, J. Skea, E. Calvo Buendia, V. Masson-Delmotte, H.-O. Pörtner, D. C. Roberts, P. Zhai, R. Slade, S. Connors, R. van Diemen, M. Ferrat, E. Haughey, S. Luz, S. Neogi, M. Pathak, J. Petzold, J. Portugal Pereira, P. Vyas, E. Huntley, K. Kissick, M. Belkacemi und J. Malley. IPCC (In press).

Jahn, T. 2013. Theorie(n) der Nachhaltigkeit? Überlegungen zum Grundverständnis einer Nachhaltigkeitswissenschaft. In Perspektiven nachhaltiger Entwicklung - Theorien am Scheideweg. Beiträge zur sozialwissenschaftlichen Nachhaltigkeitsforschung, Hrsg. J. C. Enders und M. Remig, 47-64. Marburg: Metropolis.

Jahn, T., M. Bergmann, und F. Keil. 2012. Transdisciplinarity: Between mainstreaming and marginalization. Ecological Economics 79:1-10.

Laclau, E. 2002. Emanzipation und Differenz. Wien: Turia + Kant.

Landrigan, P. J., J. L. Sly, M. Ruchirawat, E. R. Silva, X. Huo, F. Diaz-Barriga, H. J. Zar, M. King, E. H. Ha, K. A. Asante, H. Ahanchian, und P. D. Sly. 2016. Health consequences of environmental exposures: Changing global patterns of exposure and disease. Annals of Global Health 82:10-19.

Meadows, D., D.H. Meadows, E. Zahn, und P. Milling. 1972. Die Grenzen des Wachstums. Bericht des Club of Rome zur Lage der Menschheit. Stuttgart: Deutsche Verlagsanstalt.

Merleau-Ponty, M. 1966. Phänomenologie der Wahrnehmung. Berlin: de Gruyter.

Newbold, T., L. N. Hudson, S. L. L. Hill, S. Contu, I. Lysenko, R. A. Senior, L. Borger, D. J. Bennett, A. Choimes, B. Collen, J. Day, A. De Palma, S. Diaz, S. Echeverria-Londono, M. J. Edgar, A. Feldman, M. Garon, M. L. K. Harrison, T. Alhusseini, D. J. Ingram, Y. Itescu, J. Kattge, V. Kemp, L. Kirkpatrick, M. Kleyer, D. L. P. Correia, C. D. Martin, S. Meiri, M. Novosolov, Y. Pan, H. R. P. Phillips, D. W. Purves, A. Robinson, J. Simpson, S. L. Tuck, E. Weiher, H. J. White, R. M. Ewers, G. M. Mace, J. P. W. Scharlemann, und A. Purvis. 2015. Global effects of land use on local terrestrial biodiversity. Nature 520:45-50. 
Pimm, S. L., C. N. Jenkins, R. Abell, T. M. Brooks, J. L. Gittleman, L. N. Joppa, P. H. Raven, C. M. Roberts, und J. O. Sexton. 2014. The biodiversity of species and their rates of extinction, distribution, and protection. Science 344:1246752.

Popp, A., K. Calvin, S. Fujimori, P. Havlik, F. Humpenoder, E. Stehfest, et al. 2017. Land-use futures in the shared socio-economic pathways. Global Environmental Change-Human and Policy Dimensions 42:331-345.

Schneidewind, U., und M. Singer-Brodowski. 2014. Transformative Wissenschaft. Klimawandel im deutschen Wissenschafts- und Hochschulsystem. Marburg: Metropolis.

Schneidewind, U. 2015. Transformative Wissenschaft - Motor für gute Wissenschaft und lebendige Demokratie. Reaktion auf A. Grunwald. 2015 Transformative Wissenschaft - Eine neue Ordnung im Wissenschaftsbetrieb? GAIA 24 (2):88-89.

Steffen, W., W. Broadgate, L. Deutsch, O. Gaffney, und C. Ludwig. 2015. The trajectory of the Anthropocene: The great acceleration. Anthropocene Review 2:81-98.

Steffen, W., J. Rockstrom, K. Richardson, T. M. Lenton, C. Folke, D. Liverman, C. P. Summerhayes, A. D. Barnosky, S. E. Cornell, M. Crucifix, J. F. Donges, I. Fetzer, S. J. Lade, M. Scheffer, R. Winkelmann, und H. J. Schellnhuber. 2018. Trajectories of the earth system in the Anthropocene. Proceedings of the National Academy of Sciences of the United States of America 115:8252-8259.

Sutton, M. A., O. Oenema, J. W. Erisman, und A. Leip. 2011. Too much a good thing. Nature 472:159-161.

WBGU. 2011. Welt im Wandel - Gesellschaftsvertrag für eine Große Transformation 2. Veränderte. Berlin: WBGU.

Blättel-Mink, Birgit, Prof. Dr., Professur für Soziologie mit dem Schwerpunkt Industrieund Organisationssoziologie am Fachbereich Gesellschaftswissenschaften der GoetheUniversität Frankfurt am Main.

https://www.fb03.uni-frankfurt.de/soziologie/bblaettel-mink

Hickler, Thomas, Prof. Dr., Leiter der Arbeitsgruppe Biogeographie und Ökosystemforschung am Senckenberg Biodiversität und Klima Forschungszentrum (SBiK-F), Frankfurt am Main. Professur für Quantitative Biogeographie am Institut für Physische Geographie der Goethe-Universität, Frankfurt am Main.

https://www.senckenberg.de/de/institute/sbik-f/ag-biogeographie/ 
Open Access Dieses Kapitel wird unter der Creative Commons Namensnennung 4.0 International Lizenz (http://creativecommons.org/licenses/by/4.0/deed.de) veröffentlicht, welche die Nutzung, Vervielfältigung, Bearbeitung, Verbreitung und Wiedergabe in jeglichem Medium und Format erlaubt, sofern Sie den/die ursprünglichen Autor(en) und die Quelle ordnungsgemäß nennen, einen Link zur Creative Commons Lizenz beifügen und angeben, ob Änderungen vorgenommen wurden.

Die in diesem Kapitel enthaltenen Bilder und sonstiges Drittmaterial unterliegen ebenfalls der genannten Creative Commons Lizenz, sofern sich aus der Abbildungslegende nichts anderes ergibt. Sofern das betreffende Material nicht unter der genannten Creative Commons Lizenz steht und die betreffende Handlung nicht nach gesetzlichen Vorschriften erlaubt ist, ist für die oben aufgeführten Weiterverwendungen des Materials die Einwilligung des jeweiligen Rechteinhabers einzuholen. 